\title{
Novel adaptation of a method to assess responsiveness of bronchial segments in vitro
}

\author{
B.E. Mc Parland*, P.R.A. Johnson*, C.L. Armour**, J.L. Black*
}

Novel adaptation of a method to assess responsiveness of bronchial segments in vitro. B.E. Mc Parland, P.R.A. Johnson, C.L. Armour, J.L. Black. CERS Journals Ltd 1998.

ABSTRACT: In vivo agonist delivery to the lungs is characterized by absorption through the mucosal layer followed by access to the smooth muscle. When agonists are applied to perfused bronchial segments, a difference in potency to agonists applied to the serosal (outside) or mucosal (inside) surface can be demonstrated. In order to elucidate this effect in canine bronchial segments, we adapted a method to assess responsiveness of agonists applied to the inside or outside surface of canine bronchial segments.

A $2 \mathrm{~cm}$ "fluid-tight" length of bronchus was cannulated and mounted in a perfusion chamber. Auxotonic contraction of the bronchus displaced fluid inside the segment up a column and the change in height of fluid within the column (afterload) was measured as a change in hydrostatic pressure (volumetric). We assessed the optimal conditions for measuring bronchial responsiveness to acetylcholine.

Neither stretching segments lengthways from 100 to $140 \%$ of resting length, nor altering the transmural pressure from 3 to $21 \mathrm{cmH}_{2} \mathrm{O}$ had a significant effect on the potency of acetylcholine applied to the outside surface. Both acetylcholine $(n=7)$ and methacholine $(n=4)$ were approximately 10 -fold more potent when applied to the outside surface than the inside surface of bronchial segments $(p<0.0001)$. Furthermore, mechanical removal of the epithelium $(n=6)$ led to a 67 -fold increase in potency of acetylcholine applied to the inside surface compared with segments with epithelium.

The advantages that this system has over previously reported methods include: 1) agonists can be injected at a low flow rate so as to minimize epithelial stress; 2) changes in luminal volume are measured under conditions of no flow; 3) after-load can be varied such that contraction is either practically isotonic or auxotonic; and 4) a "barrier effect" of the epithelium of canine bronchial segments can be demonstrated. This new adapted method will provide us with the means to assess the relationship between in vitro and in vivo responsiveness in human bronchi. Eur Respir J 1998; 11: 1248-1256.
Depts of *Pharmacology and **Pharmacy, University of Sydney, Sydney, Australia

Correspondence: B.E. Mc Parland

Dept of Pharmacology

University of Sydney

Sydney

NSW 2006

Australia

Fax 61293513868

Keywords: Acetylcholine

dogs

epithelium

methacholine

muscle-smooth

Received: May 261997

Accepted after revision February 151998

This project was funded by the Nationa Health and Medical Research Council of Australia and B. Mc Parland by an Australian Postgraduate Award.
Airway hyperresponsiveness (AHR) is a characteristic feature of asthma. If in vivo responsiveness is based on smooth muscle responsiveness, then there should be a relationship between in vivo and in vitro responsiveness. However, no study has demonstrated within the same patient, a positive correlation between in vivo responsiveness and in vitro reactivity to histamine $[1,2]$ or methacholine $[3,4]$. There may be a methodological explanation for these findings. During a bronchial challenge, an agonist is inhaled and gains access to the smooth muscle by passing through the mucosal layer. However, in bronchial ring, strip or spiral preparations, which were used in previous studies [1-4] the agonist gains access to the smooth muscle via the exposed cut edge of the tissue, through the serosal (outside) and mucosal (inside) surface. Therefore, if the mode of agonist application in vitro is to "mimic" in vivo agonist delivery, then in vitro reactivity should be assessed by adding an agonist to the inside surface of bronchi.

Several methods have been developed that allow independent delivery of agonist to either the outside or inside surface of the bronchi and thus allow an estimate of the "barrier effect" of the epithelium to an agonist applied to its surface [5-9]. In studies using one of these, the airway narrowing analyser, a significant difference in porcine tissue responsiveness was demonstrated when acetylcholine was applied to the inside as compared to the outside of a bronchial segment [9]. However, there are several disadvantages associated with the use of this method. Firstly, the flowmeter necessitates the use of a high flow rate of fluid through a bronchial segment, and this may directly cause disruption to the epithelium. Secondly, measurement of flow rate by the flowmeter is not directly related to changes in resistance within the bronchial segment [10]. Thirdly, contraction occurs isotonically, so elastic load-ing of the segments is not possible. Initially, we used the "airway narrowing analyser" [6] to measure in vitro res-ponsiveness. When we were unable to detect a consistent difference in segment responsiveness between inside and outside application of the agonist (barrier effect), we adapted a volumetric method $[11,12]$ to allow for agonist delivery to the inside of a bronchial segment and the 
measurement of changes in luminal volume. The volumetric method allows shortening of the smooth muscle against an increasing load, i.e. auxotonic measurements and this situation more closely mimics in vivo bronchial constriction. We have defined the optimal parameters for its use in canine bronchial segments and tested the hypothesis that the barrier effect would be present in control segments with epithelium intact and absent in segments denuded of epithelium.

\section{Materials and methods}

\section{Lung collection and preparation of bronchial segments}

Dogs were killed with an overdose of barbiturate (thiopentone sodium, $\left.160 \mathrm{mg} \cdot \mathrm{kg}^{-1}\right)$. The left lung was resected and placed into Krebs-Henseleit buffered solution (Krebs) at $4^{\circ} \mathrm{C}$. All bronchial branches were ligated with silk suture to produce a bronchial segment that was "fluid tight" throughout its length. Segments were usually about $2 \mathrm{~cm}$ long. For the volumetric experiments, the main bronchial branch was cut into two segments: a large internal diameter (ID) segment and a small ID segment. The large segment was estimated to be 4-5 $\mathrm{mm}$ ID at the proximal end and $2-3 \mathrm{~mm}$ ID at the distal end. The small segment was estimated to be $2-3 \mathrm{~mm}$ ID at its proximal end and 1-2 $\mathrm{mm}$ ID at the distal end. Only large segments were used for the "airway narrowing analyser" experiments.

\section{Method I (airway narrowing analyser)}

Protocol. Both ends of a large bronchial segment were attached to adaptors and placed into a $50 \mathrm{~mL}$ horizontal organ-bath (fig. 1). The transmural pressure inside the segment was set at $5 \mathrm{cmH}_{2} \mathrm{O}$ since this approximated the transpulmonary pressure at functional residual capacity (FRC). This was done by setting the height of the constant water-head tube (fig. 1, part a) $5 \mathrm{~cm}$ above the bronchial segment. A flow rate of $60 \mathrm{~mL} \cdot \mathrm{min}^{-1}$ was set by adjusting the height of the spillway cup (fig. 1, part j). After setting the above parameters, the segments were equilibrated for $90 \mathrm{~min}$ in the bathing fluid $\left(37^{\circ} \mathrm{C}\right.$ Krebs, $95 \% \mathrm{O}_{2}, 5 \% \mathrm{CO}_{2}$ and $\mathrm{pH}$ 7.35). During this time, the bath fluid was changed at 15 min intervals, and the inside of the segment was perfused for $30 \mathrm{~s}$. Perfusion of the fluid through the inside of the bronchial segment was stopped, and the transmural pressure $\left(5 \mathrm{cmH}_{2} \mathrm{O}\right)$ was maintained by clamping the distal tube (fig. 1, part f). Following equilibration, flow was commenced, and an initial dose of acetylcholine $(100 \mu \mathrm{M})$ was added to the outside of the bronchial segment to test for tissue viability. When the response had reached a plateau, a complete washout of the agonist was achieved by exchange of the bathing fluid.

Measurement of bronchial contraction. A flow meter (fig. 1 , part $\mathrm{h}$ ) was used to measure changes in flow caused by the narrowing of the bronchial segment in response to methacholine. The flow meter generates an electrical signal which is proportional to the position of the magnetic float

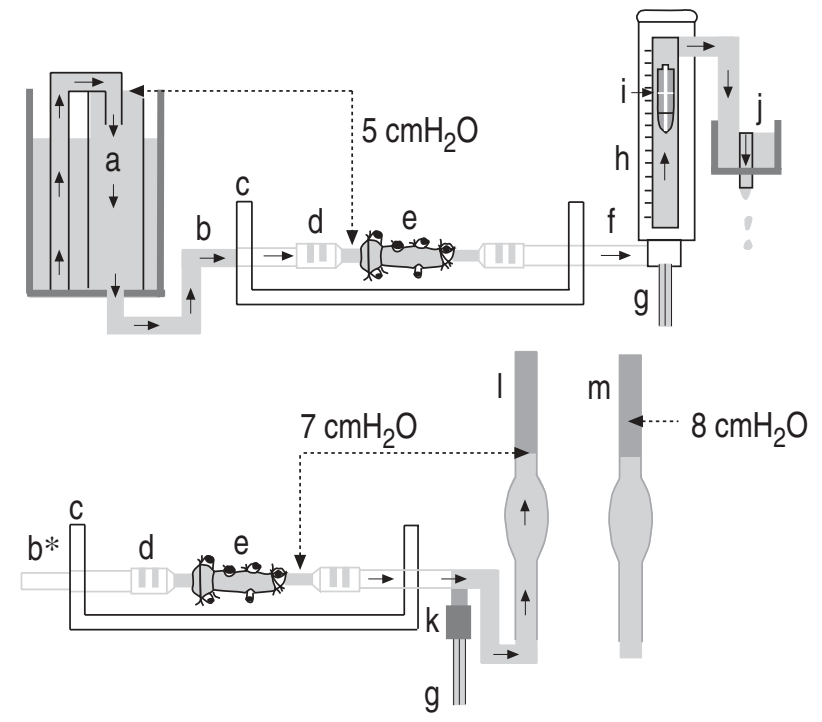

Fig. 1. - Schematic illustration of the airway narrowing analyser (top) and the volumetric apparatus (bottom). a: constant water-head tube which maintains the height of the Krebs meniscus above the bronchial segment such that the transmural pressure within the bronchial segments is 5 $\mathrm{cmH}_{2} \mathrm{O}$; b: proximal tube; $b^{*}$ : injection port which has a narrow internal diameter to minimize the fluid dead-space; c: Ugo Basile organ-bath; d: bronchial segment adaptors; e: bronchial segment; f: distal tube which is clamped to stop the flow of fluid; g: signal from flow meter or pressure transducer going to a MacLab® set up and Macintosh computer; h: flow meter (range 15-60 $\mathrm{mL} \cdot \mathrm{min}^{-1}$ ); i: magnetic float; $\mathrm{j}$ : spillway cup; k: Abbott ${ }^{\circledR}$ transpac pressure transducer; 1 : volumetric pipette showing fluid height $(7 \mathrm{~cm})$ before contractions; and $\mathrm{m}$ : fluid height $(8 \mathrm{~cm})$ after contraction of a segment.

(fig. 1, part i). The flow meter output signal was found to be linear only over a range from $120-15 \mathrm{~mL} \cdot \mathrm{min}^{-1}$, although we were able to record down to $0 \mathrm{~mL} \cdot \mathrm{min}^{-1}$. Due to the non-linearity of the flow meter at less than 15 $\mathrm{mL} \cdot \mathrm{min}^{-1}$, we performed a calibration by measuring the $\mathrm{mL} \cdot \mathrm{min}^{-1}$ of effluent from the spillway cup. A MacLab® quad bridge amplifier (ADI systems, Sydney, Australia) increased the gain of the signal, and the signal was converted from analogue to digital using a MacLab® $2 \mathrm{e}$. MacLab® chart software was used to analyse the doseresponse curves.

Influence of site of agonist application on segment responsiveness. Two large bronchial segments, from each of the nine dogs, were prepared as described earlier and placed into separate organ-baths (fig. 1, top). After equilibration and addition of the initial dose of agonist to the bronchial segments (to assess viability), methacholine was applied to the outside of the segment at increasing half log doses to produce a cumulative dose-response curve. Approximately $20 \mathrm{~min}$ after washout of the response, methacholine was perfused through the inside of the bronchial segment at increasing half log doses to produce a cumulative doseresponse curve in a manner similar to that reported in porcine bronchial segments [13]. There was no difference in our experiments for methacholine responsiveness ad-ded to either the outside or inside (fig. 2). On this basis we speculated that the high flow (baseline $30-60 \mathrm{~mL} \cdot \mathrm{min}^{-1}$ ) of perfusion fluid through the inside of the canine bronchi, as opposed to porcine [13] bronchi, increased the leakiness of the epithelium and thus increased access of 


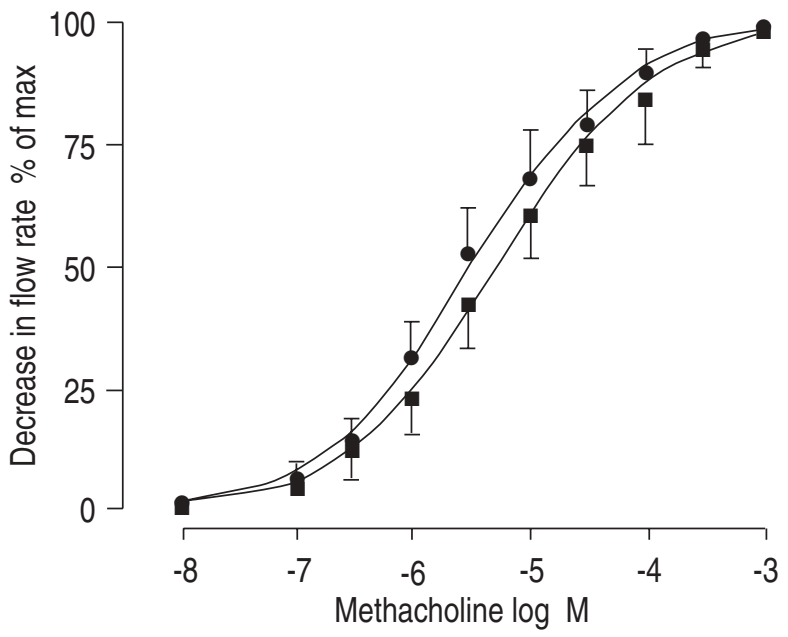

Fig. 2. - Dose-response curves to methacholine applied to the outside $(\bullet)$ or inside (ם) surface of large bronchi using the airway narrowing analyser. Data points are means \pm SEM; $n=9$ dogs (18 tissue segments).

methacholine to the smooth muscle. In all subsequent experiments, we therefore used the adapted volumetric technique which would not require continual flow for the measurement of bronchial segment contraction.

\section{Method 2 (volumetric system)}

Segments were dissected, mounted into the organ-bath and equilibrated using the same protocol as for the airway narrowing analyser. However, inside washes were achieved by perfusing via the proximal cannula $4 \mathrm{~mL}$ of Krebs $\left(37^{\circ} \mathrm{C}, \mathrm{pH} 7.35\right)$ at a rate of $2 \mathrm{~mL} \cdot \mathrm{min}^{-1}$. This slow rate was used to minimize abrasion and shear stress to the epithelium. These washes to the inside of the segment were performed every 10-15 min. Acetylcholine and methacholine responsiveness were assessed in two ways: 1) Agonists were added cumulatively at half log doses to the outside of the segment with no washout in between doses. 2) Agonists were added to the inside of the segment as a single bolus dose with a complete agonist washout period before each increasing dose of agonist. This method was used because it was technically difficult to perform cumulative dose-response curves on the inside of the bronchial segments. Although both acetylcholine and methacholine were investigated, acetylcholine was the agonist chosen for all subsequent experiments because responses could be washed out much more quickly than with methacholine.

\section{Measurement of bronchial contraction (volumetric system).}

With this new system, we measured the volume of fluid that was displaced from the inside of the segment during bronchial contraction, i.e. volumetric measurements. Fluid displacement led to an increase in fluid height in the column (increase in afterload) that was dependent upon the bore size of the column. The changes in height of the fluid were detected as changes in hydrostatic pressure with the use of an Abbott ${ }^{\circledR}$ transpac 3 pressure transducer (Abbott, Sydney, Australia). The signal from the transducer was recorded using the MacLab® system. Changes in hydrostatic pressure were recorded as $\mu \mathrm{V}$ and calibrated to both $\mathrm{cmH}_{2} \mathrm{O}$ and volume $(\mu \mathrm{L})$.

Calibration of the equipment and calculation of the volume of luminal fluid displaced. Three or four known volumes of Krebs were injected through the proximal cannula with a cannulated segment in place. The distal cannula car-ried the displaced perfusate through a column to give three or four increasing column heights. The column heights were plotted against the volume of injected Krebs, and a best-fit equation was generated (fig. 3). To calculate the volume of luminal fluid displaced as a result of outside application of an agonist, the gradient from the best fit equation was multiplied by the change in column height. For inside responses, the height of the column after injection of a known volume was multiplied by the gradient and the constant added ( $c=-156 \mu \mathrm{L}$, fig. 3 ) to give the total volume response. The difference between the total volume response and the known volume injected was the volume of luminal fluid displaced due to contraction. The volume injected for inside additions of an agonist was approximately five times the internal volume of the segment. This volume was injected at a slow rate of $2 \mathrm{~mL} \cdot \mathrm{min}^{-1}$, such that the increased volume did not constitute a pressure load. When a range of volumes was used, the change in luminal volume due to narrowing of the bronchial segment was identical. The volume injected was sufficient to displace approximately $90 \%$ of the original fluid within the segment. This was verified by the injection of trypan blue through the inside, followed by the withdrawal of $100 \mu \mathrm{L}$ of luminal fluid and comparison of its optical density with that of the pre-injected trypan blue.

Reproducibility of the volumetric method. The reproducibility of responsiveness to outside administration was assessed by the comparison of two consecutive cumulative dose-response curves to acetylcholine (dose range 10 $\mathrm{nM}-10 \mathrm{mM} ; \mathrm{n}=6$ dogs). The reproducibility of responsiveness to inside administration was assessed by the comparison of two consecutive bolus dose-response curves to

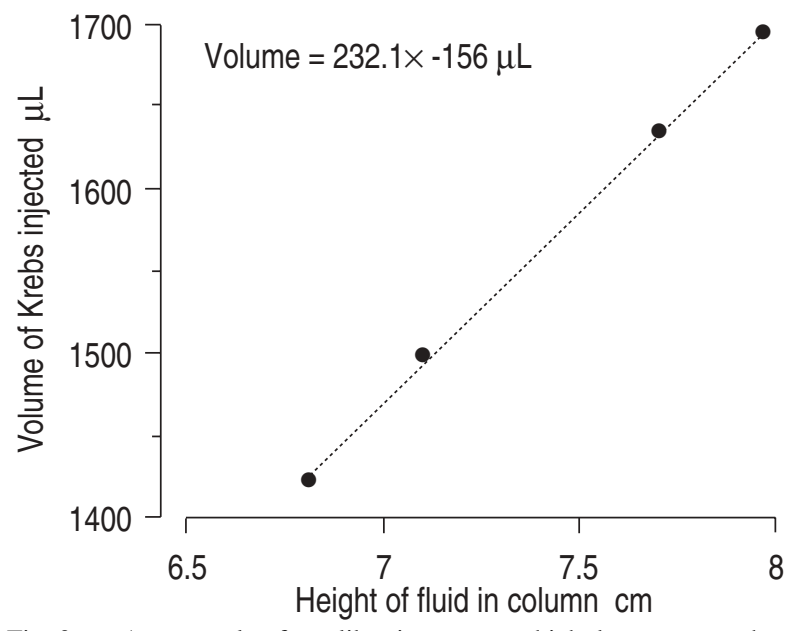

Fig. 3. - An example of a calibration curve, which demonstrates that a change in column height (pressure) is directly proportional to the volume of Krebs injected into the lumen. 
acetylcholine (dose range $0.1 \mu \mathrm{M}-10 \mathrm{mM} ; \mathrm{n}=3$ dogs). In order to normalize responses to acetylcholine, a single maximal bolus dose $(10 \mathrm{mM})$ of a more efficacious agonist (methacholine) was added to the outside of a bronchial segment at the end of a dose-response curve. Methacholine, rather than acetylcholine, was used since more than $10 \mathrm{mM}$ acetylcholine would have been required for a maximal response, and this produced a significant alteration in the osmolarity of the bath fluid.

Comparison of cumulative and bolus administration. In a separate series of experiments, a comparison was made between responses obtained by cumulative and bolus administration to the outside. In each bronchial segment, a cumulative dose-response curve was performed, and this was followed by a bolus dose-response curve ( $n=3$ dogs).

Experiments to determine optimal transmural pressure (preload). The fluid height in the column governs the transmural pressure/radial smooth muscle length within the segment preparation. To determine the optimal transmural pressure of segments at resting length, the transmural pressure was randomly set at $0,3,7,11,16$ or 21 $\mathrm{cmH}_{2} \mathrm{O}$ and dose response curves were performed on the outside surface.

Estimation of optimal stretched length. To determine the optimal stretched length of the tissue, bronchial segments were placed in the organ bath at resting unstretched length $(100 \%$, i.e. $2.0 \mathrm{~cm})$ with a constant transmural pressure of $7 \mathrm{cmH}_{2} \mathrm{O}$, and then bronchial length was increased in $10 \%$ $(0.2 \mathrm{~cm})$ increments until it was $140 \%(2.8 \mathrm{~cm})$ of its initial resting length. Segments were equilibrated for $15 \mathrm{~min}$ at their new stretched length, and a full dose-response curve to acetylcholine on the outside surface was performed ( $n=3$ dogs).

Influence of site of agonist application and the influence of bronchial size on responsiveness. Small and large bronchi in these experiments were stretched to $120 \%$ of resting length and the transmural pressure was set at $7 \mathrm{cmH}_{2} \mathrm{O}$. Dose-response curves to acetylcholine were performed on the outside and inside surface for both small and large bronchi ( $\mathrm{n}=7$ dogs). Comparisons were made between inside and outside responses to acetylcholine for small and large segments.

Removal of the epithelium. To determine the influence of an intact epithelium, dose-response curves were performed on tissues with the epithelium mechanically removed. Removal of the epithelium was achieved by passing the bronchial segment over soft dental floss (four threads of 2 $\mathrm{mm}$ diameter Oral B super floss $\AA$ ) that was tied to a fixed object at one end, with the other end inserted through the inside of the segment. Using the same method, our group has shown that the proportion of epithelium remaining after denudation was approximately $20 \pm 3 \%$ [14].

A comparison using methacholine (a more stable choline ester). Any observed difference in potency of acetylcholine applied to the inside or the outside of the bronchus may be the result of increased acetylcholinesterase activity on the inside, which leads to a lower potency, and thus the difference in potency should be either abolished or at least decreased when methacholine is used. Methacholine is more resistant than acetylcholine to metabolism by acetylcholinesterase. In order to test this, dose-response curves to methacholine (half log doses) were performed on the outside and inside surface for both small and large bronchi ( $\mathrm{n}=3$ and 4 dogs, respectively). Comparisons were made between inside and outside responses to methacholine for small and large segments.

Solutions, chemicals and equipment. Solutions and chemicals: Krebs-Henseleit solution, $\mathrm{pH} 7.35$ (composition in mM: $\mathrm{NaCl}, 118 ; \mathrm{KCI}, 4.7 ; \mathrm{CaCl}_{2}, 2.5 ; \mathrm{MgSO}_{4}, 1.2 ; \mathrm{NaH}_{2} \mathrm{PO}_{4}$, 1.2; $\mathrm{NaHCO}_{3}, 25.5$; D-glucose, 11.1). Krebs solution was continually gassed with $95 \% \mathrm{O}_{2}$ and $5 \% \mathrm{CO}_{2}$. The agonists used were: acetylcholine chloride and methacholine (Sigma Chemicals, St. Louis, MO, USA). Agonists were dissolved in deionized water and serially diluted with Krebs solution on the day of the experiment. The agonists were kept on ice throughout the experiment.

Equipment: silk suture (Cyanamid, Sydney, Australia Pty Ltd); Abbott@ transpac 3 pressure transducer, cat \#L 9691 (Abbott ${ }^{\circledR}$, Sydney, Australia); Organ bath and airway narrowing analyser (Ugo Basile, Italy); MacLab® 2e and MacLab® bridge amplifier (ADI instruments, Sydney, Australia).

Statistical methods. Results were expressed as means \pm SEM. Contraction (decrease in luminal volume) was expre-ssed as a percentage of the maximum obtainable contraction caused by $10 \mathrm{mM}$ methacholine added to the outside surface. Values for - $\log \mathrm{EC}_{50}$ (negative $\log$ dose which gives a $50 \%$ of maximum decrease in luminal volume) obtained at different bronchial lengths and transmural pre-ssures were expressed as means \pm SEM. A dose ratio (DR) was derived from the - $\log$ EC50 values to compare differences between inside and outside application of agonist. ANOVA and Fishers protected least-squares difference test were used to detect differences between curves and between -logEC50 values. Significance was defined as pð 0.05 in all cases.

Ethical approval. The Animal Experimentation Ethics Com-mittee (AEEC) of the University of Sydney approved our request to obtain canine airway tissue from dogs that had been killed with an overdose of barbiturate.

\section{Results}

\section{Method 1 (airway narrowing analyser)}

Site of agonist application. Dose-response curves to methacholine applied to the outside surface were not significantly different from those generated from addition to the inside surface of large bronchi (fig. 2) (n=9).

\section{Method 2 (volumetric system)}

Reproducibility. Second cumulative dose-response curves on the outside surface for both small and large bronchi 
a)

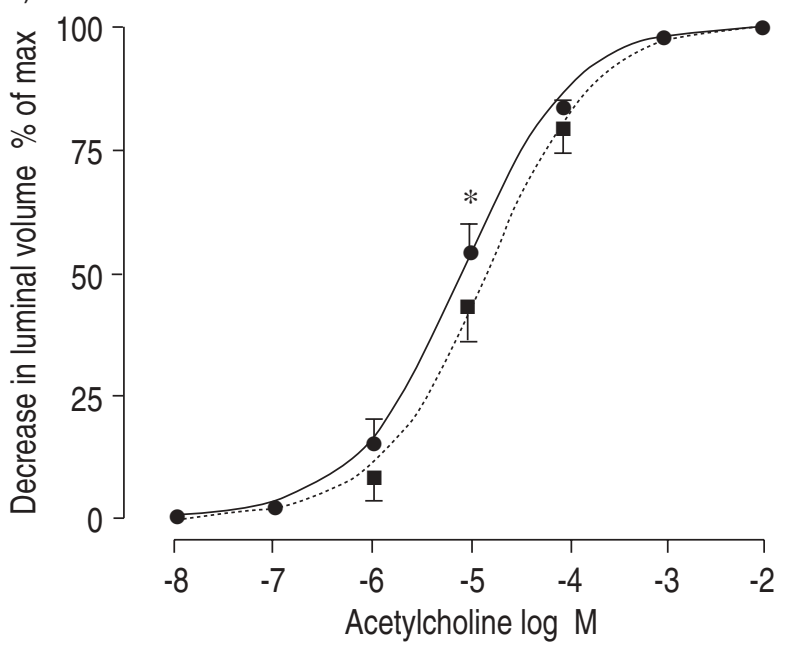

b)

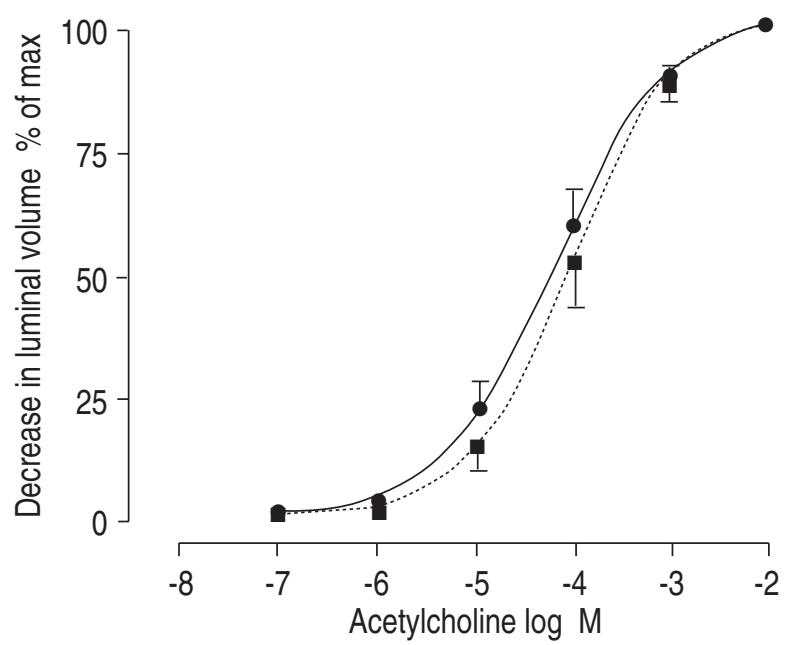

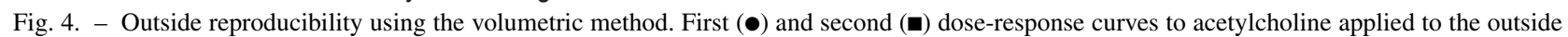
surface of a) small or b) large bronchial segments. Solid and broken lines represent curves of best fit for first and second dose-response curves, respectively. Data points are means \pm SEM; $n=6$ dogs. *: significant difference (pð0.05).

(fig. 4a and $\mathrm{b}$ ) were not significantly different from the first dose-response curves ( $n=6)$. In addition, second doseresponse curves on the inside surface for both small and large bronchi (fig. 5a and b) were also not significantly different from the first dose-response curves $(n=3)$.

Comparison of cumulative and bolus administration. Bolus dose-response curves on the outside surface of large bronchi (fig. 6) were not significantly different from cumulative dose-response curves $(n=3)$.

Optimal transmural pressure (preload). In both small and large bronchi, increasing the transmural pressure to 3 $\mathrm{cmH}_{2} \mathrm{O}$ and above caused a significant $(\mathrm{p}<0.05, \mathrm{n}=3$ and 4 dogs, respectively) leftward shift in the dose-response curve to acetylcholine (increased sensitivity) compared with responses elicited at a pressure of $0 \mathrm{cmH}_{2} \mathrm{O}$. How-

a)

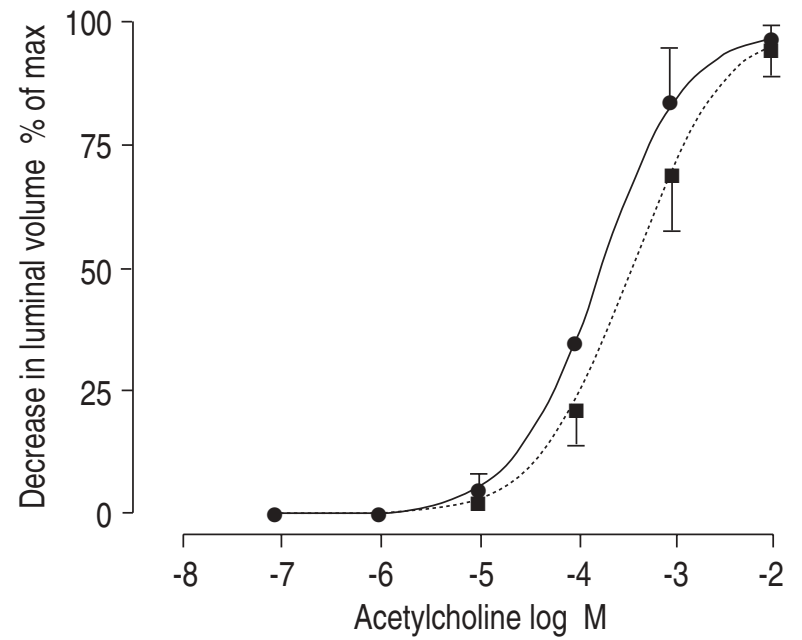

ever, from 3-21 $\mathrm{cmH}_{2} \mathrm{O}$, there were no significant differences in potency of acetylcholine (fig. 7a). The maximum volume of luminal fluid displaced for both small and large segments (maximum response) increased with transmural pressures $0-16 \mathrm{cmH}_{2} \mathrm{O}$ (fig. 7b). At $16 \mathrm{cmH}_{2} \mathrm{O}$, small segments reached a maximum response, however, for large segments, the maximum response may not have been attained, even at $21 \mathrm{cmH}_{2} \mathrm{O}$ (fig. 7b). The change in volume of the small segments as the pressure was increased (fig. 7b) was greater than for the large segments, indicating that the former were more compliant.

Optimal stretched length of bronchi. Stretching bronchi from resting unstretched length to a maximum of $140 \%$ had no significant effect on dose-response curves to acetylcholine applied to the outside surface of either small or large bronchi (fig. 8).

b)

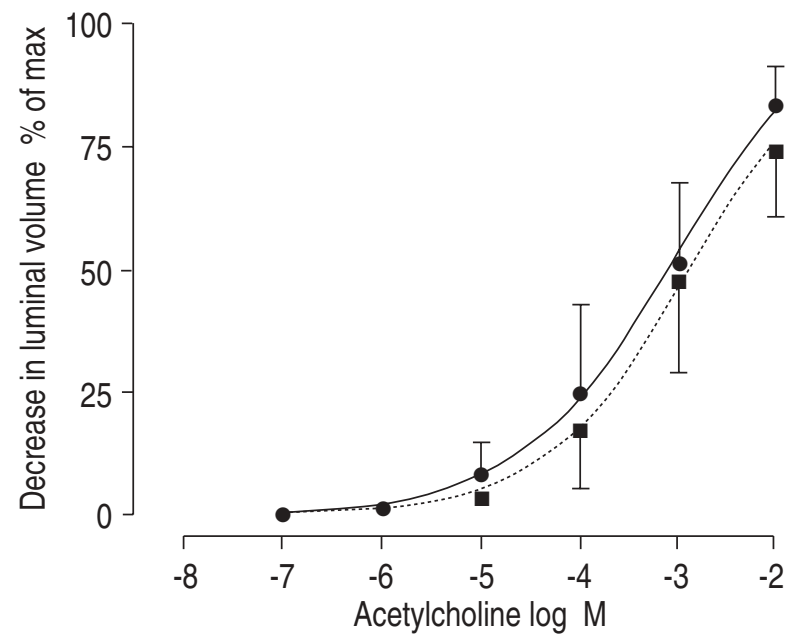

Fig. 5. - Inside reproducibility using the volumetric method. First $(\bullet)$ and second ( $\bullet$ ) dose-response curves to acetylcholine applied to the inside surface of a) small or b) large bronchial segments. Solid and broken lines represent curves of best fit for first and second dose-response curves, respectively. Data points are means \pm SEM; $n=3$ dogs. *: significant difference (pð0.05). 


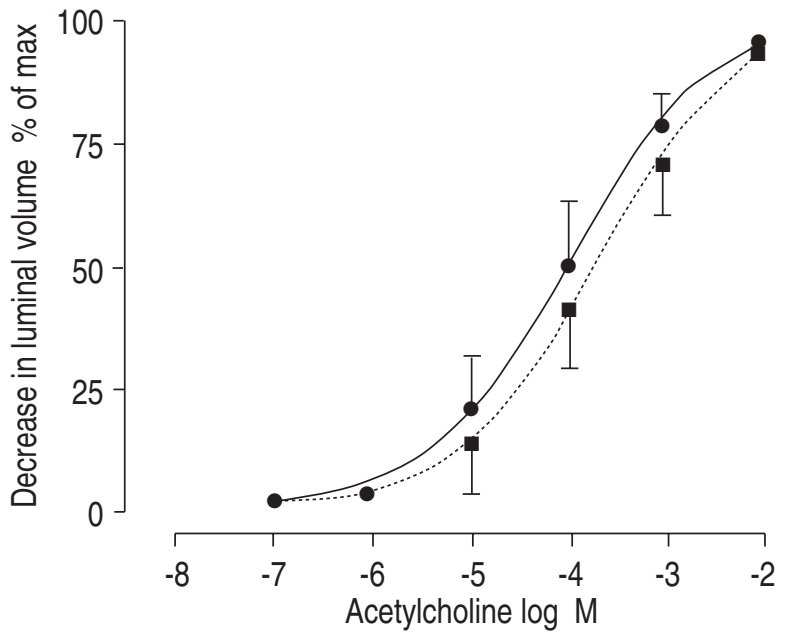

Fig. 6. - Cumulative versus bolus administration of acetylcholine to the outside of large bronchial segments using the volumetric method. Cumulative ( $\bullet$ ) and bolus ( $\boldsymbol{\square})$ dose-response curves for large bronchi ( $\mathrm{n}=3$ dogs). Results expressed as means \pm SEM.

Site of agonist application and bronchial size. Dose-response curves to acetylcholine applied to the outside surface of segments stretched to $120 \%$ of their initial length and at a transmural pressure of $7 \mathrm{cmH}_{2} \mathrm{O}$, were significantly $(\mathrm{p}<0.0001, \mathrm{n}=7)$ shifted to the left of the dose-response curves generated from addition of acetylcholine to the inside surface in both small (fig. 9a) and large (fig. 9b) bronchi. Acetylcholine was six-fold more potent when applied to the outside surface of small bronchi, than the inside (table 1). In large bronchi, acetylcholine was 10fold more potent when applied to the outside than the inside surface (table 1); By comparing figure 9a with figure $9 \mathrm{~b}$ the effect of bronchial size can be inferred. The sensitivity to acetylcholine applied to the outside of small segments (fig. 9a) was greater than for large segments (fig. 9b). Acetylcholine was five fold more potent when app-

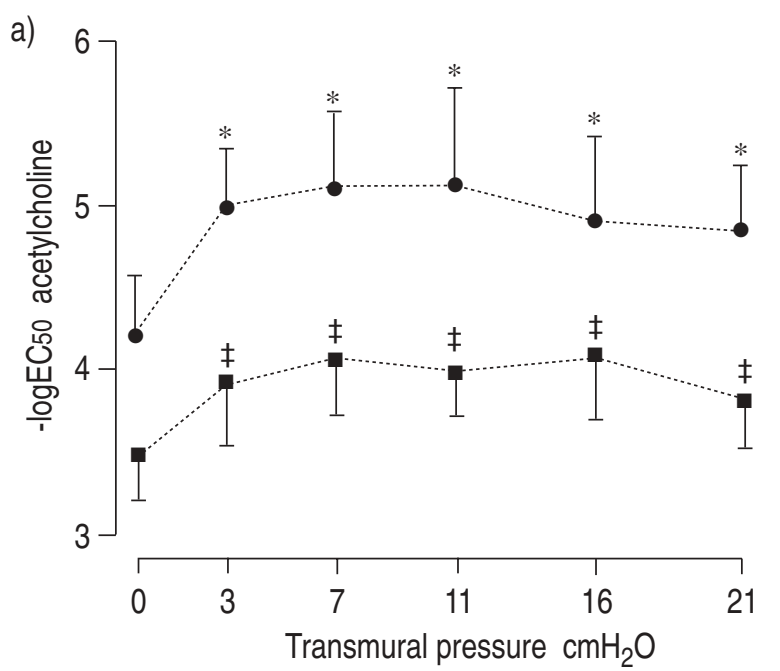

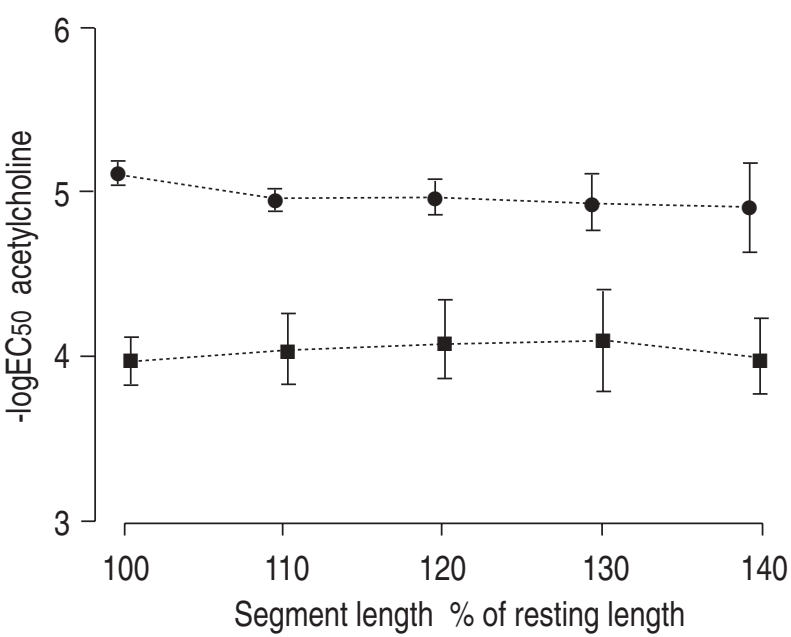

Fig. 8. - The effect of stretching small (•) and large (๘) bronchi lengthways on responsiveness to acetylcholine applied to the outside surface while using the volumetric method ( $n=3$ dogs). Results expressed as mean negative log dose which gives a $50 \%$ of maximum decrease in luminal volume $(-\log E C 50)$ values \pm SEM.

lied to the outside of small compared with large bronchi (table 1). Acetylcholine was 8-fold more potent when applied to the inside of small compared with large bronchi (table 1).

Removal of the epithelium. When the epithelium was removed, the dose-response curve to acetylcholine applied to the inside surface was significantly shifted to the left compared to when the epithelium was intact (fig. $9 \mathrm{~b}, \mathrm{n}=6$ and 7 , respectively). Acetylcholine applied to the inside surface of large bronchial segments denuded of epithelium was 63-fold more potent than when applied to the inside surface of large bronchial segments with the epithelium intact.

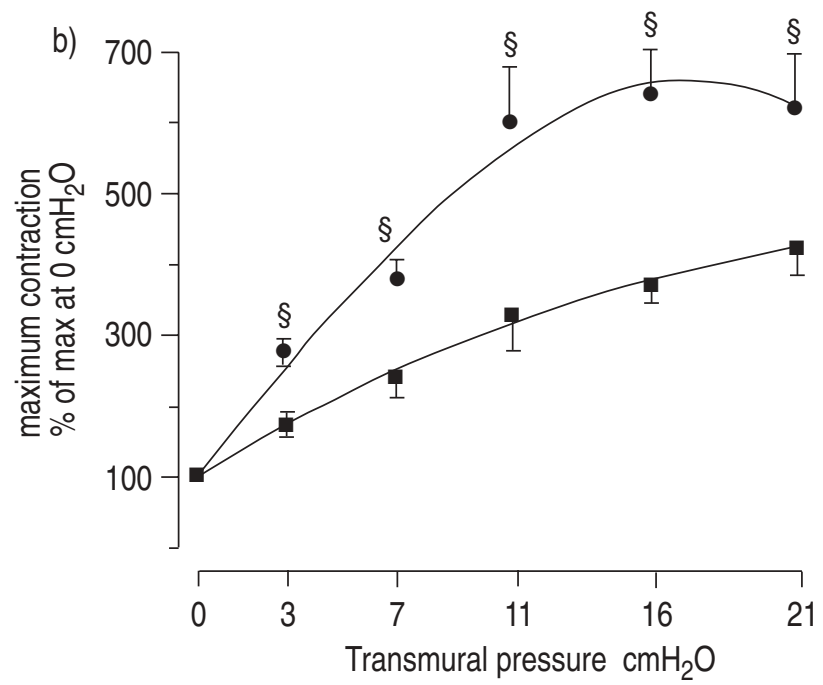

Fig. 7. - a) Effect of transmural pressure on the potency of acetylcholine when applied to the outside of small $(\bullet)$ and large $(\boldsymbol{\square})$ segments $(\mathrm{n}=3$ and $\mathrm{n}=4$ dogs, respectively) using the volumetric method. Results expressed as mean -logEC50 values \pm SEM. * and $¥$ : significantly (pð0.05) greater negative $\log$ dose which gives a $50 \%$ of maximal decrease in luminal volume (-logEC50) when compared with the -logEC50 value obtained at a transmural pressure of $0 \mathrm{cmH}_{2} \mathrm{O}$ for small and large bronchial segments, respectively. b) The effect of transmural pressure on the maximum response to acetylcholine applied to the outside surface of small $(\bullet)$ and large ( $\bullet$ segments $(\mathrm{n}=4 \mathrm{dogs})$. Results expressed as means \pm sEM. All points are significantly different from the value obtained at $0 \mathrm{cmH}_{2} \mathrm{O}$ transmural pressure. The relationship between transmural pressure and the maximum contraction (percentage of maximum at $\left.0 \mathrm{cmH}_{2} \mathrm{O}\right)$ to acetylcholine is significantly different $(\S: \mathrm{p}<0.05)$ when small and large bronchi are compared. 
a)

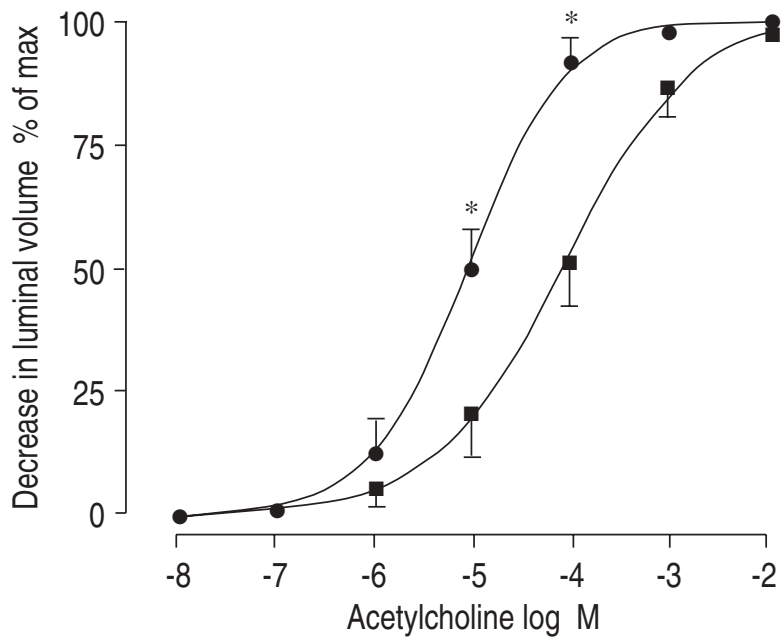

b) 100

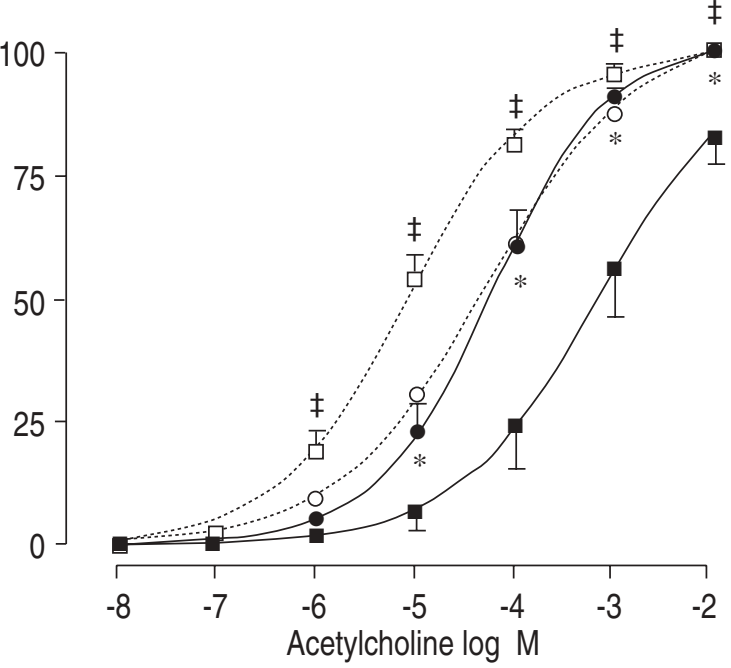

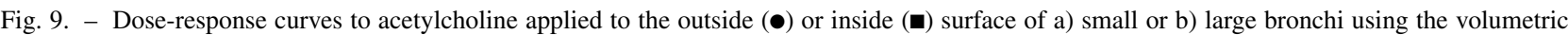
method. Open symbols represent responses to acetylcholine applied to the outside $(\bigcirc)$ or inside $(\square)$ surface of large segments denuded of epithelium. Data points are means \pm SEM; $n=7$ with intact epithelium and $n=6$ denuded of epithelium. *: significant difference (pð0.05) between inside data points and outside data points. segments denuded of epithelium.

Table 1. - The -logEC50 values obtained from outside and inside application of acetylcholine or methacholine for both small and large bronchial segments using the volumetric method

\begin{tabular}{lccccc}
\hline $\begin{array}{l}\text { Size of } \\
\text { preparation }\end{array}$ & Agonist & $\mathrm{n}$ & Outside & Inside & DR \\
\hline Small & Acetylcholine & 7 & $5.0 \pm 0.2$ & $4.2 \pm 0.2$ & 6 \\
Large & Acetylcholine & 7 & $4.3 \pm 0.2$ & $3.3 \pm 0.3$ & 10 \\
Large, denuded & Acetylcholine & 6 & $4.3 \pm 0.2$ & $5.1 \pm 0.1$ & 0.16 \\
Large* & Methacholine & 9 & $5.4 \pm 0.2$ & $5.2 \pm 0.3$ & 2 \\
Small & Methacholine & 3 & $6.5 \pm 0.3$ & $5.2 \pm 0.1$ & 20 \\
Large & Methacholine & 4 & $5.8 \pm 0.2$ & $4.7 \pm 0.4$ & 13
\end{tabular}

A - $\operatorname{logEC} 50$ value was obtained for each individual bronchial segment, and then a mean for all experiments was derived. DR (dose ratios) are given to demonstrate the relative difference in potency to acetylcholine applied to different surfaces and - $\operatorname{logEC} 50$ values are expressed as means_SE. *: results obtained using the airway narrowing analyser.

a)

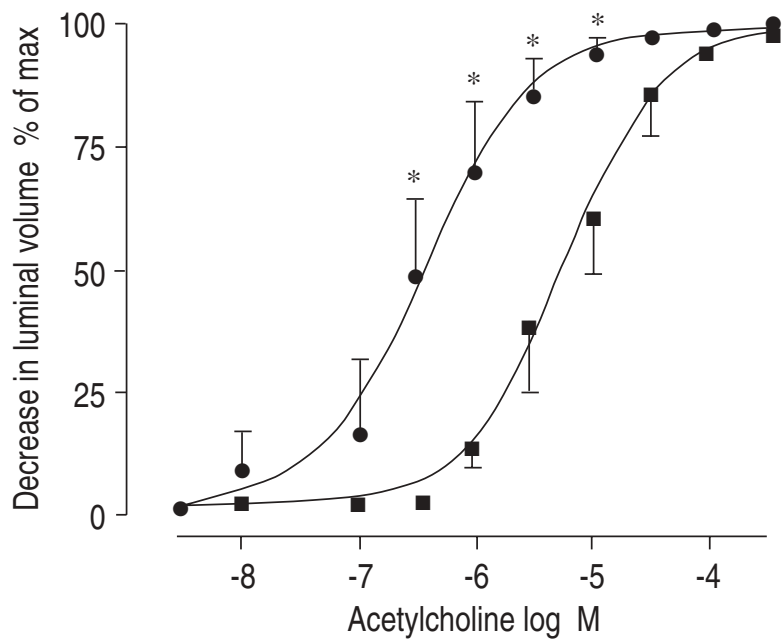

Use of methacholine. Dose response curves to methacholine applied to the outside surface were significantly $(\mathrm{p}<$ $0.0001, \mathrm{n}=3$ and 4 dogs, respectively) shifted to the left of the dose response curves generated from addition of methacholine to the inside surface in both small (fig. 10a) and large (fig. 10b) bronchi. Methacholine was 20- and 13fold more potent when applied to the outside, compa-red to the inside, surface of small and large bronchi, res-pectively (table 1).

\section{Discussion}

We have adapted and evaluated a volumetric method for measurement of airway hyperresponsiveness in canine bronchial segments in vitro. We have defined the optimal parameters for its use and established that a "barrier effect" of the epithelium can be demonstrated in our adapted system in vitro.

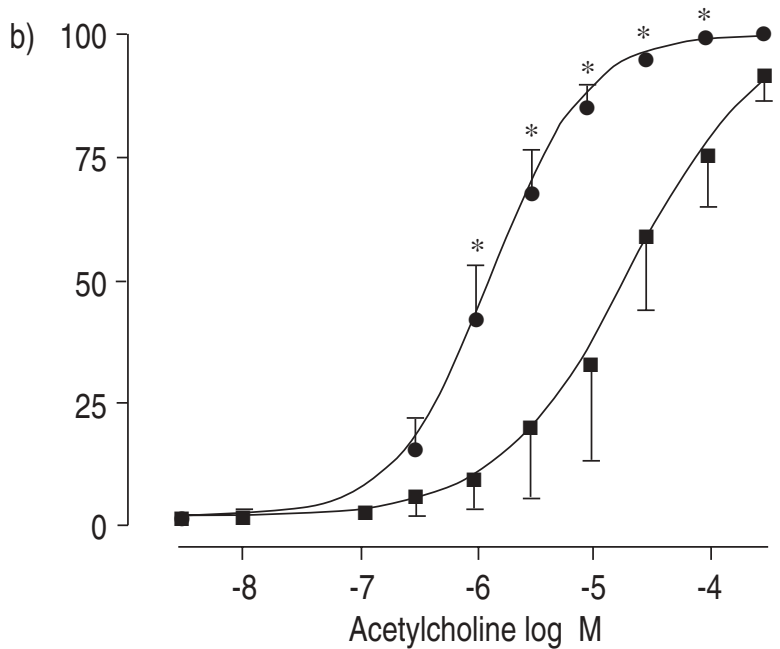

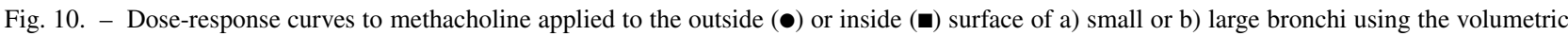
method. Data points are means \pm sEM; $n=3$ and $n=4$ for small and large segments, respectively. *: significant difference (pð0.05) between inside and outside data points. 
What makes our system novel is that we have combined the method of measurement used by MARTIN and PROCTOR [11] and Moreno et al. [12] with a perfusion system based on the method of Mitchell and Sparrow [13]. Unlike the former, we were, therefore, able to assess responses to drugs applied to both inside and outside of segments. Unlike the latter, we were able to measure contraction auxotonically, and we were able to dispense with the high flow rates at the time of agonist injection. The high flow rate with the use of the "airway narrowing analyser" method of Mitchell and Sparrow [13] seemed to affect the "epithelial barrier" of canine bronchi in our study, although not porcine in their experiments [13].

During smooth-muscle shortening, the luminal fluid within the bronchial segment is displaced up a column, and the increase in column height during contraction increases the load against which the segment has to constrict (afterload). Therefore, the volumetric technique allows shortening of the bronchial smooth muscle fibres against an increasing load, and this is termed auxotonic contraction, which is most probably the way in which bronchi contract in vivo [15-17]. During inspiration, bronchial smooth muscle fibres shorten against an increasing elastic load. Therefore, at higher lung volumes, the elastic loading is greater than at low lung volumes. With greater elastic loading, the airways become less responsive to inhaled methacholine [18]. This new system not only provides us with a method whereby the different effects of an agonist added to the inside or outside of a bronchial segment can be observed, but also provides a technique where the magnitude of an elastic load can be changed by altering the diameter of the column and its effects on smooth-muscle shortening of a bronchial segment measured. For the present study, changes in afterload have been minimized by using a large bore column.

Our results demonstrated that the potency of acetylcholine applied to the outside surface of bronchial segments was not altered by stretching bronchial segments longitudinally. This result was not surprising, since there is no evidence in canine bronchi that contraction has a longitudinal component [19] and in rabbit bronchi the longitudinal component is small [20]. Since bronchial responsiveness did not vary at lengths from 100-140\% of initial unstretched resting length, the stretched length of $120 \%$ (midpoint) was chosen as the parameter setting for all subsequent experiments. At $120 \%$ stretched length, the luminal volume of the segment was about $20 \%$ greater than at resting length, and this meant that the changes in luminal volume during contraction were greater and thus were measured more accurately.

In this study, a wide range of transmural pressures (3$21 \mathrm{cmH}_{2} \mathrm{O}$ ) could be used without a significant difference in the potency $(-\log E C 50)$ of acetylcholine applied to the outside surface. This result may appear to be surprising. However, potency may not be a good indicator of lengthtension relationships, since it is calculated from a pharmacological maximum which may occur at, or before, closure of a bronchial segment. The maximum response to $1 \mathrm{mM}$ methacholine significantly increased with increasing transmural pressure (fig. 7b), and results based on maximum response may therefore provide a better indicator of length-tension relationships. Small airways reached a maximum response at $16 \mathrm{cmH}_{2} \mathrm{O}$ transmural pressure, and there was no decrease in the maximum response for large bronchi at all transmural pressures used. Furthermore, from these results, it was deduced that small segments were more compliant than large segments. This difference may explain why the optimal transmural pressure (11-21 $\mathrm{cmH}_{2} \mathrm{O}$ ) for small segments was less than that for large segments ( $\breve{\mathrm{S}} 21 \mathrm{cmH}_{2} \mathrm{O}$ ), since small segments would have stretched to optimal operating length at a lower pressure than for large segments.

Our most important criterion in developing the novel volumetric system was that a "barrier effect" to an agonist applied inside the lumen could be demonstrated. In contrast to results obtained using the airway narrowing analyser, we have demonstrated that both methacholine and acetylcholine were approximately 10 -fold less potent when applied to the inside surface than when applied to the outside surface of canine bronchial segments. It has been suggested that the attenuated responses on the inside compared with outside responses are caused by a greater cholinesterase activity on the inside surface, however, this is unlikely since the difference in potencies was similar for both acetylcholine and methacholine (which is more resistant to hydrolysis by cholinesterases). The possibility also exists that the difference in potency was the result of comparison between bolus dose administration performed on the inside and cumulative dose administration performed on the outside. This is unlikely, since we showed that, in the same segment, there was no difference in responses obtained using the two different methods of administration (fig. 6).

Upon removal of the epithelium, the dose-response curve to acetylcholine applied to the inside surface was shifted leftward from the control dose-response curve in the presence of an intact epithelium. The potency of acetylcholine applied to the inside surface of denuded segments increased approximately 60-fold compared with control segments with epithelium. Removal of the epithelium did not alter responses to acetylcholine applied to the outside surface. From this study, we are unable to deduce the mechanism by which the epithelium decreases the potency of an agonist applied to its surface. Interestingly, acetylcholine was sixfold more potent on the inside compared with the outside surface of bronchial segments denuded of epithelium. This difference between inside and outside potency of acetylcholine may be the result of a greater perfusion distance and thus a lower concentration of agonist at the level of the smooth muscle for outside application than for inside application in these experiments.

Previous studies using porcine bronchial segments have shown that segments with a small internal diameter are more responsive to acetylcholine applied to the outside surface than segments with a large internal diameter. The effect of internal diameter of a bronchial segment on responsiveness to acetylcholine and methacholine was also observed using our adapted method. In small segments, both acetylcholine and methacholine were fivefold more potent than in large bronchial segments when applied to the outside surface. This effect has not been observed with bronchial ring, strip or spiral preparations, but does occur when bronchial segments are used and responses are measured isometrically (tension without changes in smooth muscle length) or isotonically (changes in length without an increasing load) [21]. This effect of a lower observed potency in large segments compared with small segments 
may be a result of a thicker adventitia and thus a greater gradient of agonist at the level of the smooth muscle [21, 22]. Similarly, small internal diameter segments were more responsive than large segments to inside application of acetylcholine and methacholine. An epithelium more permeable to acetylcholine in small bronchi compared with large bronchi could explain the greater potency of acetylcholine applied to the inside of small bronchi than of large bronchi [23]. Another possibility is that a thicker mucosal layer leads to a greater gradient of agonist and thus a lower observed potency in large internal diameter segments compared with small segments [21, 22].

The present study has demonstrated that with the use of this adapted technique, which measures changes in luminal volume, a difference in agonist potency with inside and outside application of methacholine and acetylcholine was observed and was abolished upon removal of the epithelium. However, there was no difference in agonist potency with inside and outside application of methacholine when the airway narrowing analyser was used for canine bronchial segments. This new adapted method will provide us with the means to assess the relationship between in vitro and in vivo responsiveness in human bronchi.

Acknowledgements: We are grateful for the supply of canine lung from T. Campbell and his technical staff from the Garvin Institute of Medical Research, St Vincent's Hospital. We are also thankful for equipment/software supplied by Abbott ${ }^{\circledR}$ Australia, Cyanamid Australia and ADI Instruments Australia.

\section{References}

1. Vincenc KS, Black JL, Yan K, Armour CL, Donnelly PD, Woolcock AJ. Comparison of in vivo and in vitro responses to histamine in human airways. Am Rev Respir Dis 1983; 128: 875-879.

2. Armour CL, Lazar NM, Schellenberg RR, et al. A comparison of in vivo and in vitro human airway reactivity to histamine. Am Rev Respir Dis 1984; 129: 907-910.

3. Roberts JA, Raeburn D, Rodger IW, Thomson NC. Comparison of in vivo airway responsiveness and in vitro smooth muscle sensitivity to methacholine in man. Thorax 1984; 39: 837-843.

4. Armour CL, Black JL, Berend N, Woolcock AJ. The relationship between bronchial hyperresponsiveness to methacholine and airway smooth muscle structure and reactivity. Respir Physiol 1984; 58: 223-233.

5. Gao YS, Vanhoutte PM. Lowering $\mathrm{PO}_{2}$ induces epithelium-dependent relaxation in isolated canine bronchi. Am J Physiol 1989; 257: C1034-C1037.

6. Mitchell HW, Willet KE, Sparrow MP. Perfused bronchial segment and bronchial strip: narrowing $v s$. isometric force by mediators. J Appl Physiol 1989; 66: 2704-2709.
7. Munakata M, Huang I, Mitzner W, Menkes H. Protective role of epithelium in the guinea pig airway. J Appl Physiol 1989; 66: 1547-1552.

8. Hulsmann AR, Raatgeep HR, Bonta IL, Stijnen T, Kerrebijn KF, de Jongste JC. The perfused human bronchiolar tube characteristics of a new model. J Pharmacol Toxicol Methods 1992; 28: 29-34.

9. Omari TI, Sparrow MP. Epithelial disruption by proteases augments the responsiveness of porcine bronchial segments. Clin Exp Pharmacol Physiol 1992; 19: 785-794.

10. McFawn PK, Sparrow MP, Mitchell HW. Measurement of flow through perfused bronchial segments: optimization of flow head resistance. Eur Respir J 1995; 8: 161-166.

11. Martin HB, Proctor DF. Pressure-volume measurements in dog bronchi. J Appl Physiol 1958; 13: 337-343.

12. Moreno RH, Hogg JC, McLean TM, Pare PD. Isovolumetric and isobaric rabbit tracheal contraction in vitro. $J$ Appl Physiol 1987; 62: 82-90.

13. Sparrow MP, Mitchell HW. Modulation by the epithelium of the extent of bronchial narrowing produced by substances perfused through the lumen. Br J Pharmacol 1991; 103: 1160-1164.

14. Black JL, Johnson PRA, McKay KO, Carey D, Armour CL. Levcromakalim and isoprenaline-induced relaxation of human isolated airways - role of the epithelium and of $\mathrm{K}^{+}$channel activation. Pulm Pharmacol 1994; 7: 195-203.

15. Stephens NL, Seow CY, Halayko AJ, Jiang H. The biophysics and biochemistry of smooth muscle contraction. (Review). Can J Physiol Pharmacol 1992; 70: 515-531.

16. Macklem PT. Theoretical basis of airway instability. Roger S. Mitchell Lecture. Chest 1995; 107: 87S-88S

17. Ishida K, Pare PD, Blogg T, Schellenberg RR. Effects of elastic loading on porcine trachealis muscle mechanics. $J$ Appl Physiol 1990; 69: 1033-1039.

18. Ding DJ, Martin JG, Macklem PT. Effects of lung volume on maximal methacholine-induced bronchocons-triction in normal humans. J Appl Physiol 1987; 62: 1324-1330.

19. Shioya T, Munoz N, Leff A. Effect of resting smooth muscle length on contractile response in resistance airways. J Appl Physiol 1987; 62: 711-717.

20. Opazo-Seaz A, Okazawa M, Paré P. Airway smooth muscle (ASM) orientation and airway stiffness favor airway narrowing rather airway shortening. Am Rev Respir Dis 1994; 49: A583.

21. Gray PR, Mitchell HW. Effect of diameter on force generation and responsiveness of bronchial segments and rings. Eur Respir J 1996; 9: 500-505.

22. Mitchell HW, Sparrow MP. Increased responsiveness to cholinergic stimulation of small compared to large diameter cartilaginous bronchi. Eur Respir J 1994; 7: 298305.

23. Hulsmann AR, Raatgeep HR, Denhollander JC, Bakker WH, Saxena PR, de Jongste JC. Permeability of human isolated airways increases after hydrogen peroxide and poly-L-arginine. Am J Respir Crit Care Med 1996; 153: 841-846. 